\title{
24
}

\section{Aboriginal transitions research project in British Columbia, Canada}

\author{
Sarah Cormode
}

\section{Context}

The project was initiated by the University of Victoria, Office of CommunityBased Research (OCBR), the University of Victoria - Office of Indigenous Affairs (INAF) and Indigenous Adult and Higher Learning Association (IAHLA), who jointly responded to a call for proposals issued by the provincial government for research projects considering various 'transition' experiences of students on the journey to post-secondary education. The three partners proposed to jointly conduct comprehensive community-based research to investigate the transition of Aboriginal students from Aboriginal-controlled post-secondary education institutes to public post-secondary education institutes.

Aboriginal students face a number of barriers to attaining a post-secondary education in Canada. As Hunt-Jinnouchi et al. (2009) note, 'the Learning Journey has been fraught with challenges for many Aboriginal students due to the impacts of generations attending residential school, racism, discrimination and the lack of meaningful, relevant cultural curriculum' (p. 17). Aboriginal-controlled adult learning institutes can play an important role in beginning to redress the disparity between Aboriginal and non-Aboriginal educational achievement in particular, as high school graduation is a significant determinant of transition to future study. When Aboriginal students are supported to complete high school, the disparity between Aboriginal and non-Aboriginal student graduation rates virtually disappears (Mendelson, 2006). This finding is supported by the current study (HuntJinnouchi et al., 2009) that notes 'completion, or near completion of previous programmes at Aboriginal-controlled [learning] institutes, is a barometer for success' (p. 38).

Further, Aboriginal-controlled learning institutes can assist students beyond the attainment of a high school diploma. They offer a culturally supportive environment in which the unique needs of Aboriginal learners are recognized, they help Aboriginal students prepare for the challenges of leaving their home community to pursue further education, and can help students navigate complex university systems and requirements. Despite such a vitally important role, relatively little is known about the role these institutes play in helping students successfully transition to post-secondary education. 
Lastly, in the words of one project participant: 'Our survival as distinct nations will depend on our ability to control our own educational institutions, so that they are used as a tool to strengthen identity and culture, rather than as a tool for assimilation and cultural genocide' (ibid., p. i). Aboriginal-controlled learning institutes play an important role in strengthening Aboriginal communities and culture.

The project was designed to address this gap in the existing research by asking students and administrators in Aboriginal-controlled learning institutes these basic research questions: for those that have transitioned, what helped you? For those that will transition in the future, what will help you? What role did your Aboriginal-controlled institute play in this transition? The project sought to better understand the barriers and success factors in supporting transition to post-secondary education and propose a number of recommendations to better support student transitions.

\section{Organization/structure}

This project is governed by a steering committee of representatives from the Offices of Community-Based Research and Indigenous Affairs at the University of Victoria, as well as representatives from the Indigenous Adult and Higher Learning Association and the project manager.

A project research team, consisting of senior administrators from the University of Victoria, IAHLA representatives, a project manager, research coordinator, administrative assistant and seven research assistants has overseen the research process itself. Members of this group met throughout the project to discuss key decisions, such as: the development of research agreements; the ethics application process; development of the research design; development of the research questions; orientation of research assistants; and the developing analysis. This group was instrumental to the project and helped to keep the project rooted in community. For example, Sarah Cormode recalled that it was the suggestion of representatives from the IAHLA institutes that the project employ communitybased researchers rather than UVic graduate students to complete the data collection. This was an important innovation to the project for a number of reasons - it helped to ensure the project was welcomed into the community, provided for richer data and has helped build capacity in the local communities with whom this project is working. This group also reviewed any draft findings and analysis before the project was finalized.

Lastly, local representatives in each community played an important role in ensuring that cultural and community protocols were respected, helping research assistants connect with the appropriate people and setting up interviews.

\section{Activities}

From the beginning, conversation between project partners was identified as important to the research process. This meant agreeing on key principles, collaboratively 
drafting research questions, ensuring that a research agreement was drafted in a language that made sense in community, and working collectively with the community-based researchers to analyse the data.

The project adopted a community-based participatory research framework and employed several methods, including a literature review, interviews and focus groups for data collection. This way of working together was chosen because it is reflective of the values that partners bring to the project and is also an important acknowledgment of cultural values important to project partners. In the words of Pauline Waterfall, 'this process was a respectful cultural interpretation, based on our traditional ways of doing and being - to embrace the individual and bring it back to the collective' (Hunt-Jinnouchi et al., 2009, p. 70).

For example, data was analysed by the community researchers using a thematic analysis, an approach that was considered important because it provides for on-going analysis throughout the research process ... [and is] consistent the important concept of a holistic worldview fundamental to First Nations' (ibid., p. v). This process also allows for the continual inclusion of new material as it is identified and can help ensure that the research remains responsive to community needs. Culturally relevant practice was important to the research process in other ways too. For example, of the practice of opening project meetings in prayer, Cormode says this helped to ground the group in the collective, to remind partners that this project was collectively owned by all involved.

\section{Outcomes}

In many regards, the actual outcomes have exceeded partners' initial expectations - support for, and participation in the project was higher than originally anticipated; the quality of data collected is 'richer' than initially imagined; and the ongoing participation of all parties has helped to ensure that project outcomes are relevant and impactful.

This project has validated the role of Aboriginal-controlled learning institutes and has added to a growing body of theory that discusses Aboriginal student success. It has made an important contribution to this dialogue from the perspectives of Aboriginal people themselves, at a time when many public post-secondary institutions are struggling with questions of how to support Aboriginal student success. Fundamentally, this project encourages a shift in the ways that these issues are addressed - from a model that has traditionally seen low Aboriginal completion rates as the result of a 'deficit' on the part of Aboriginal students themselves, to a new understanding that it is post-secondary (and other mainstream) institutions that need to change to support Aboriginal student success. Projects such as these support post-secondary institutions to make these changes.

This project has also made an important contribution to the theory and practice of community-based research, generally. According to Cormode, projects such as these can 'demonstrate a respectful way of doing research in communities and for the benefit of communities' and are an important reminder that there is no 'one size fits all' approach to community-based research. 
Participation in this project has had a number of direct impacts on the lives of participants. For the community researchers, participation in the project had an empowering effect. Many of the community researchers continue to be involved in dialogue around this project and several have become involved in other research projects. For Cormode, their participation in this project helped to provide a foundation for their continued involvement with this type of work.

Another important impact for participants occurred through the process of documenting their experiences. Participation was, for many students, an emotional process. Several noted how important it was to them to be given an opportunity to discuss the challenges and frustrations they face in returning to school, as well as to celebrate their successes. Expressing and documenting these experiences can be an important part of the healing process for Aboriginal students, many of whom have experienced the damaging effects of the residential schools system and all of whom have experienced the daily effects of colonization.

Equally important was the role that documentation played in the research with staff and administrators at the IAHLA institutes. Participation in this project helped document the importance of cultural practices in the classroom and to provide a forum for staff and administrators to share their experiences, challenges and successes.

Lastly, for public post-secondary institutions, this project has contributed to an increased understanding of 'where their students are coming from' and appropriate supports to ensure their success.

This project has many other impacts, most notably the legacy it leaves behind. While funding will run out after 2012, participation in this project has supported the development of new relationships between IAHLA members and between IAHLA institutes and public post-secondary institutions. For example, Cormode cited a recent open forum event, hosted by the project, as a place where networking and relationship building occurred. The feedback received indicates there is a real need for these types of opportunities and that there is interest among participants in continuing to forge stronger relationships.

Further, the project has potential to leave an important legacy through the pilot projects that it funded in its second year. These projects, focused on testing out several of the key project recommendations, will have an impact beyond their immediate participants and will result in the development of key resources for use by IAHLA institutes and public post-secondary institutions.

Lastly, the cultural and social impact of projects such as these cannot be overstated. These projects help broaden understanding of issues that Aboriginal students face and help to challenge stereotypes and misconceptions about these students. Culturally, this type of project supports the need for culturally appropriate practices at public post-secondary institutions, and there is some evidence to suggest that this message is being heard. Cormode cites the 2010 opening of the First Peoples' House at the University of Victoria as an example of the growing recognition of the role of culture in supporting Aboriginal student success (web. uvic.ca/fphouse). Another example of the cultural impact of this project can been found in one of the Transformative Indigenous Learning Strategies pilot projects 
partially funded through the project. This pilot gives students the opportunity to work one-on-one with a SENĆOFEN Elder to learn the SENĆOFEN language and receive credit towards graduation for their participation, helping to ensure the survival of the SENĆOFEN language. There is, of course, a very long way to go. 